\title{
STAR SPOTS ON AR LAC TYPE STARS
}

\author{
D. M. POPPER \\ University of California \\ Los Angeles \\ USA
}

There is no generally accepted definition of AR Lac Stars, and the term RS CVn stars is used interchangeably or to refer to a particular subgroup. For the purposes of this discussion I use the term AR Lac stars to refer to detached close binaries showing Ca II emission in at least the cooler component outside eclipse, the hotter component being a main-sequence or subgiant star of spectral type $F$ or G. Most of the systems show irregularities in their light curves as well as period changes. In order to determine whether a system is detached, one must know both the mass ratio and the relative radii. The determination of minimum masses is a fairly straightforward spectroscopic task, and provisional values are available for 22 of the systems, two or possibly three of them being non-eclipsing. All but 3 (AD Cap, RT Lac, RV Lib) have masses of the two components within $30 \%$ of each other. Because of appreciable irregularities in the light curves, the radii are subject to considerable uncertainty even when photometry of good precision is available. Nevertheless the 9 systems with very provisional radii all appear to be detached. These all have mass ratios near unity. We may assume, as a working hypothesis, that the other systems with mass ratios near unity are also detached and hence also belong in the AR Lac group. Most of the data referred to are to be found in IBVS 1083.

In a presentation before Commission 42 I will discuss the massradius and color-luminosity relations for these stars and suggest that their present condition may be ascribed to normal stellar evolutionary processes combined with a moderate amount of mass exchange, perhaps through the medium of a stellar wind enhanced in the binary system. Evidence for the flow of matter in these detached systems is provided by the appreciable period variations in a number of them and possibly by the radio flaring observed in the brightest systems. These systems are distinguished from non emission systems of similar mass by having more evolved components. It appears that the stars

Edith A. Müller (ed.), Highlights of Astronomy, Vol. 4, Part II, 397-403. All Rights Reserved. Copyright (C) 1977 by the IAU. 
tend to misbehave as they move into the Hertzsprung gap after they have depleted their core hydrogen fuel, have developed convective envelopes, and have started to evolve more rapidly.

So much for what I mean by AR Lac systems. What about starspots ? I have never published a statement or otherwise taken the position that starspots exist on these stars, and I do not know whether they exist. I don't know either why I didn't have enough sense to turn down the invitation to participate in this discussion. Potential evidence for the existence or non-existence of starspots must come primarily from photometric observations, and I am principally a spectroscopic observer. But since I am here, it behooves me to make some pertinent comments.

The potential evidence referred to lies in irregularities in the light curves of a number of these systems. There is only one set of irregularities known to me that seems to provide a measure of direct evidence for the presence of starspots in systems of this type. They are Kron's original observations of AR Lac itself, during the partial phases of primary eclipse obtained many years ago. The individual observations have been published only in graphical form. Some roughness of the light curve during these phases was attributed to the covering or uncovering of spots. To my knowledge these observations have not been repeated, nor has the effect been found in other systems. Observations of exceptionally high quality are required.

There is much more evidence on irregularities in the light curves of systems in this group outside eclipse, and I suppose it is these observations that the organizers of this discussion had in mind. By far the best studied of the systems photometrically is RS CVn, principally by virtue of the work of Catalano, Rodono, and others at Catania. Three slides are shown of their famous investigations. There have been a number of discussions of the cause of the travelling wave in the light curve of RS SVn which I do not intend to review here. There have been suggestions of such waves in a few other systems, but its presence in none of them has been as clearly established as for RS SVn. In that system the wave has been followed pretty closely for a full cycle. Will it suffer the fate of other apparently regular phenomena in variable-star astronomy and fail to perform according to prediction in the future ?

Next I would like to show a sampling of partial light curves obtained at Kitt Peak in 1972 and 1973. These are all systems, as is RS, CVn, having total eclipses. It should be emphasized that ellipticity and reflection effects should be negligible in all of them. LX Per appears to be a well behaved system at the observed epoch, but it is not distinguishable in its fundamental properties (mass, radius, state of evolution, etc.) from the other systems. Fluctuations in the light of RU Cnc, RW UMa and UX Com (Figures 1 and 2) are shown. 


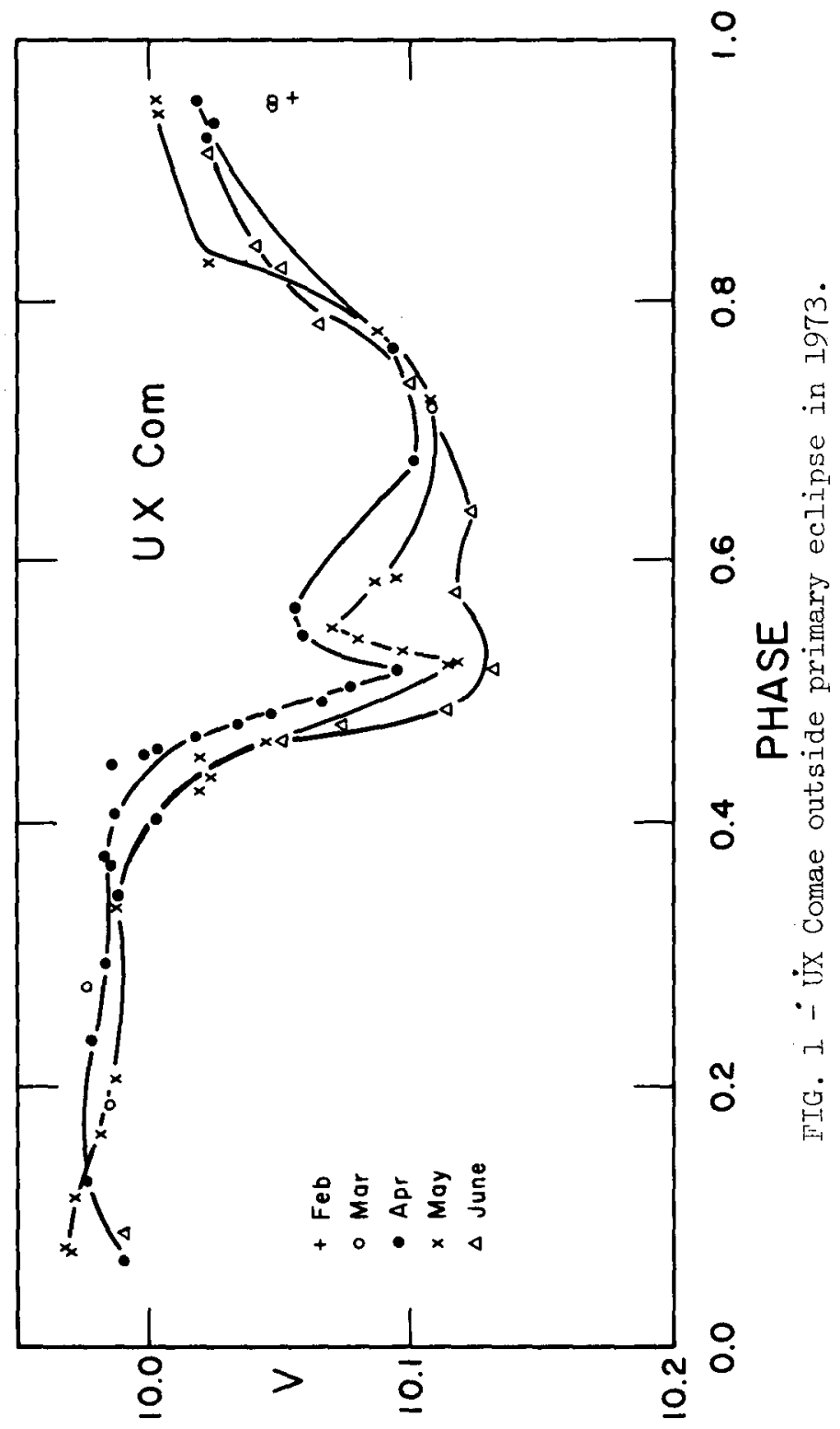




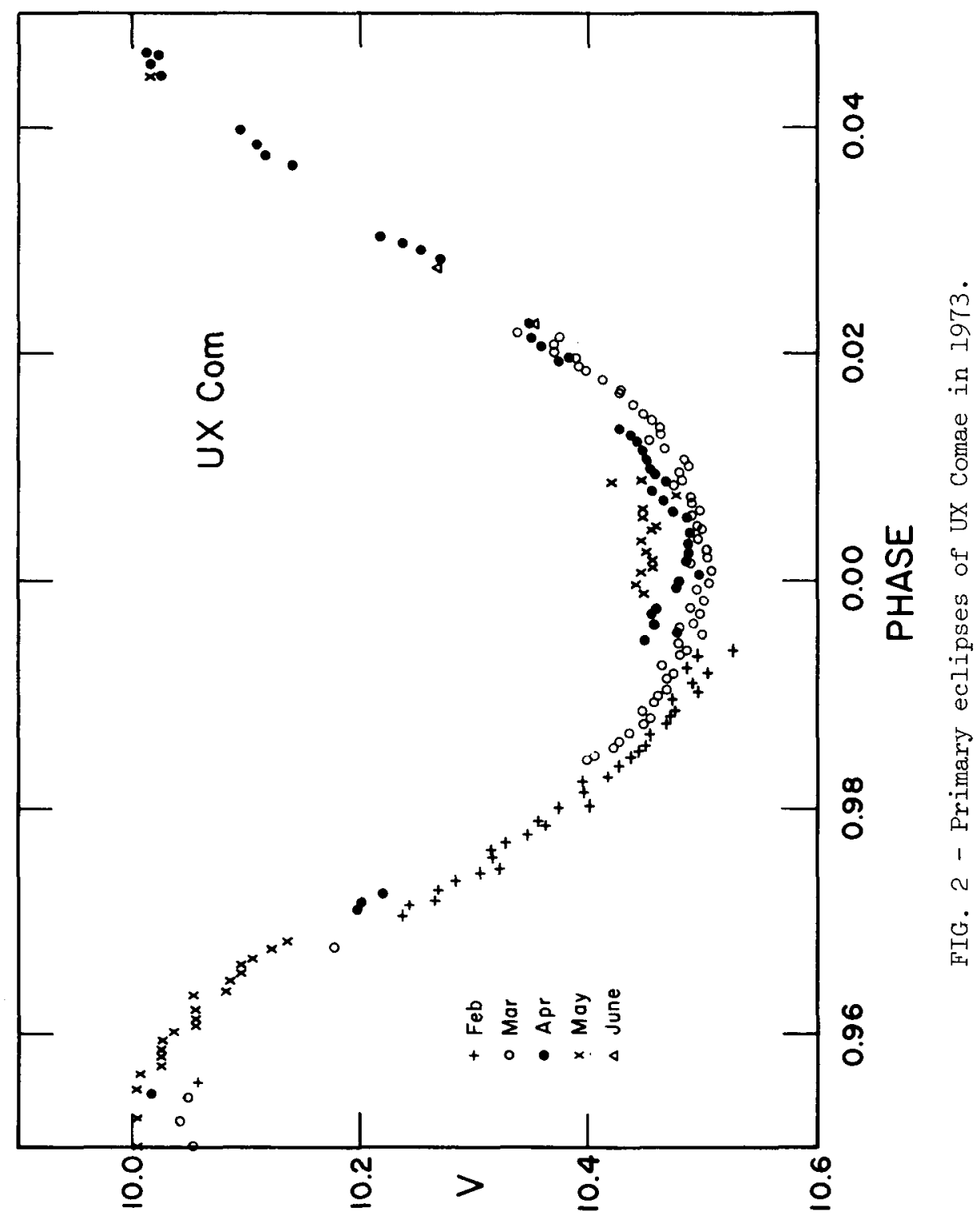


The ranges of the fluctuations in units of the light of the larger, cooler star are listed.

\begin{tabular}{lccc} 
RU Cnc & UX Com & RS CVn & RW UMa \\
\hline $15: \%$ & $15: \%$ & $30: \%$ & $30: \%$
\end{tabular}

The general form of these fluctuations in a given system persists for at least a few orbital periods, so that the fluctuations have roughly the orbital period and presumably the period of axial rotation as well. What evidence exists is in favor of equal orbital and axial periods.

To obtain some clue as to the origin of these variations we may examine color-magnitude plots for the light outside primary eclipse. In each of these four systems from my own observations (Figure 3) the system is redder when it is more luminous. This result points very strongly to the redder, larger star as the source of the variations in each system. In AR Lac itself the fluctuations are reported to be associated mainly with the hotter component. Each system needs to be observed carefully. Evidence in favor of the cooler components of the four systems as the sources of the fluctuations outside primary eclipses comes from the equality of the fluctuations in $B$ and in $V$ light when they are expressed as fractions of the light of the cooler star. Independent though less strong evidence (Figure 3) comes from the similarity in the color-luminosity relation for points outside eclipses and inside secondary eclipse. Since the two stars in a given system differ in color by only about $0.5 \mathrm{mag}$, since the light of the larger star is diluted by that of the hotter, and since the fluctuations are 15-30\% of the star, moderate changes in the color of the fluctuating star itself would cause secondorder effects, detectable only with observations of extremely high precision.

I can think of three potential ways of changing the contribution of the light from the cooler star in a reasonably neutral way: dark spots, obscuration by dust or gas, and pulsation. One can construct ad hoc models of obscuring clouds and of drifting spot groups, but the clouds would appear to be very difficult to account for dynamically, and spot groups giving up to $30 \%$ light variation may be just as difficult to support on a physical basis. With respect to pulsation, it has been usually ruled out because of the color variations expected. But large irregular light variations accompanied by only small color variations are found among variable stars (RV Tau, SR classes), perhaps caused by non-radial oscillations in more than one mode. Is it possible that we see counterparts on a smaller scale in our stars, with the oscillations excited in some way by aspects of the binary system ? I hasten to add that I do not support any particular explanation but rather 




FIG. 3 - Correlation between magnitude and color index for AR Lac systems. Filled dots: outside eclipses; open circles: within secondary eclipses. 
point out that oscillations provide a potential explanation perhaps worth investigating. I am indebted to $\mathrm{R}$. K. Ulrich for discussions on this point.

Additional observations are, of course, essential. Several of these systems need to be followed photometrically over a wide range of wavelengths. Some work by D. S. Hall and E. F. Milone has indicated modest infrared excesses. These need to be confirmed and extended. Variations in light as large as $30 \%$ should show in the relative intensities of spectrum lines. The kind of effect reported by Kron for AR Lac needs to be looked for.

In conclusion, I repeat that I do not know whether starspots are present on AR Lac stars. 